\title{
Die vier hohen Cs des Hermann Burger*
}

Enrico Danieli

* Gedanken zur Ausstellung über Hermann Burger anlässlich seines 20. Todestages am 28. Februar 2009 im Museum Strauhof in Zürich an der Augustinergasse 9 (bis am 1. März), Di-Fr 12-18 Uhr, Sa-So 10-18 Uhr.

Korrespondenz:

Dr. med. Enrico Danieli

Via ai Colli 22

CH-6648 Minusio

e.b.danieli@bluewin.ch
Früher, beinahe wäre man geneigt zu sagen, als das Wünschen noch geholfen hat, war Manie mit Genialität gekoppelt, Melancholie Basis der Denkschulen.

Erinnern wir uns an Adrian Leverkühns (erkaufte) Genialität (im Pakt mit dem Teufel Krankheit), fällt uns, vom Ende her betrachtet, seine letzte Höllenfahrt ein: sein abgeschlossenes kompositorisches Werk Apocalypsis cum figuris und sein Abschied von den Freunden: «Wachet mit mir!» Dabei ist von gottmenschlicher Not die Rede; und weiter: «Schlafet ruhig und lasset euch nichts anfechten. Verlasset mich nicht! Seid um mich zu meiner Stunde!» Verzeihen Sie mir diesen literarischen Exkurs. Schliesslich soll unser Weg zu Hermann Burger (1942-1989) und seiner Ausstellung führen. Sind wir erst am Ende des Rundganges angelangt, werden sich die Kreise schliessen. Hermann Burger, dem Sprachgenialen aus dem Aargauer Stumpenland, ist diese Gedenkausstellung gewidmet. Der Titel der Ausstellung «Nachlass zu Todeszeiten» birgt schon so viel in sich, Rätselhaftes und Biographisches, dass es sich lohnt, dem Titel nachzuhorchen. Hermann Burger (Autor bekannter Bücher wie «Schilten», «Der Schuss auf die Kanzel», «Brunsleben», «Kirchberger Idyllen» u.a.m.) war Zeit seines Lebens vom Tode umstellt. In seiner Küttiger Zeit (1972-1982) hatte er den Todesacker gleich dreifach bei sich: vor, neben und hinter dem Haus: daheim auf dem Friedhof. Und schon als Dreijährigem hat sich ihm sein Daheim eingeprägt durch Grossmutters Satz: «Do usse bisch deheime, Bueb!» In allen Büchern und Schriften des Autors wird das Leben vom Tod aus gesehen. So galt/ gilt er denn auch als Spezialist in diesem Gebiet, nannte sich selber einen Totologen. Seine Todesfixiertheit (-besessenheit?) durchzieht sein ganzes Schaffen. Eine Art von situativer (wie wir gesehen haben), aber auch von gedanklicher (wie wir sehen werden) Beengung.

Lassen Sie sich Zeit mit den Ausstellungsräumen im Erdgeschoss: Sie werden einiges aus Hermann Burgers Biographie und aus seinem Gotthardstollenbuch «Die künstliche Mutter» erfahren. Witziges, Dramatisches, Verqueres: ein guter Einstieg.

Doch dann (mit diesem Wissen vorbelastet und mit dem mulmigen Gefühl, nun auf das zu stossen, das uns medizinisch mit dem Autor über seine Zeit hinaus verbindet) werden wir hinein-

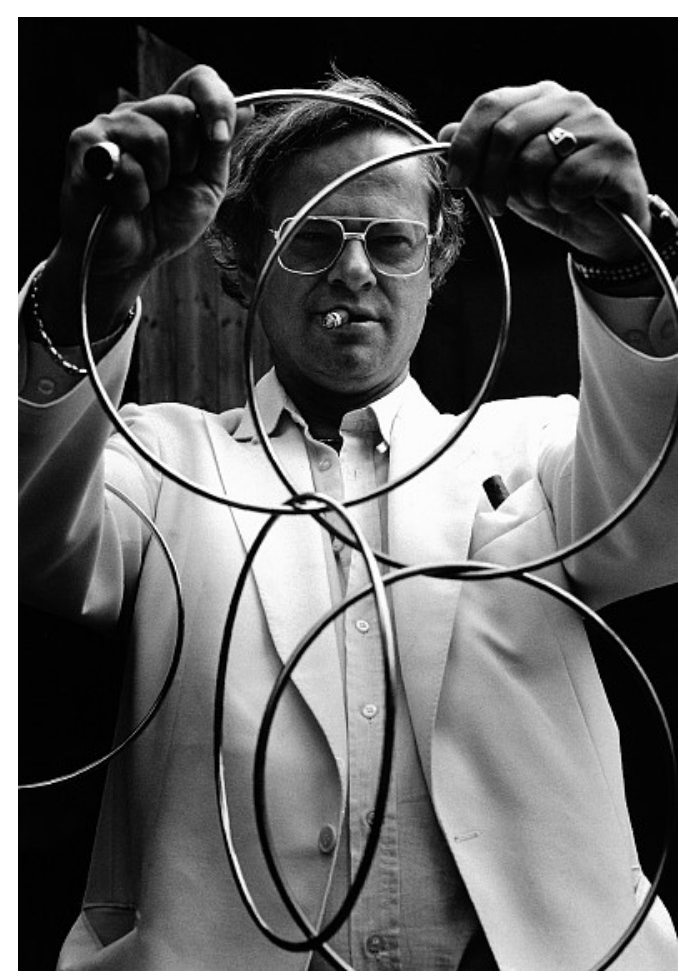

Hermann Burger mit Zauberringen.

Foto: Yvonne Böhler.

gesogen, hineingewirbelt in das, was wir - medizinisch-diagnostisch - gesucht haben: Wir sind im 2. Stockwerk angekommen und beim ersten hohen C. Burgers Friedhofhang, wie erwähnt, ist nicht erst seit «Schilten» bekannt. Das Cimiterische, seine innige Anziehung zu all den Campi santi, zu seinem «Daheim», zu allen Begräbnisund Grab- und Einäscherungsvarianten, hat ihn stets begleitet: «Diesseits der Mauer darf gelten: wer lebt, der lebe auf ewig! / Jenseits: gestorben ist tot. Keiner kam je zurück.» («Kirchberger Idyllen») Dass, wir greifen vor, dass ihn das Cimiterische mit aller Macht am Dienstag, den 28. Februar 1989 heimsucht, Burger endete seinem Tractatus logico-suicidalis entsprechend durch Suizid, nehmen wir mit Entsetzen zur Kenntnis. Die zehn Tabletten Vesparax haben ihm einen selbstgewählten Tod ermöglicht. Seine ihn beschäftigende Trias Scheintod - scheinlebendig - Verschollenheit blitzt an allen Orten, in allen Texten auf. Er wie kaum ein anderer huldigte diesem «mitten im Leben vom Tod umfangen». Burger schrieb, in Abwandlung eines berühmten Zitates: «Ich sterbe, also bin ich», ein Basso continuo, der seinem Leben und seinem 
Schreiben die Melodie der Trauermusik unterlegt. Die Cigarren, seine zweite grosse Liebe, lasse man am besten in «Band I, Brunsleben: Brenner» Revue passieren, dort (erschienen am 1. März 1989) werden wir detailversessen eingeführt in die hohe Kunst des zu Asche sich wandelnden Cigarrenrauches. «Dieses allmähliche Sicheinnebeln, dieses Gestaltwerden in den Schwaden ist für mich das Urbild schöpferischer Tätigkeit.» Für Hermann Burger war blauer Rauch ein Medium: Es diente ihm als Erinnerung, als Inspiration, aber und vor allem (auch wenn es schwerfällt, Medizinern dies mitzuteilen) als Genuss-, noch mehr als Trost-, am meisten als Heilmittel - gegen Depression. Zum dritten (hohen) C führt die Manege. Halten wir einen Moment inne im Sägemehl, geniessen, vom Zauberer Burger verführt zu werden, der diese Kunst nicht nur perfekt beschrieben, sondern auch selber gerne ausgeübt hat. Amateurmagier, Magier der weissen Kunst, war Burger in seinem fünften Nebenberuf (Privatdozent, Cigarier, Redakteur, Vibraphonist). Das Circensische bestimmte des Autors glücklichere Lebensstunden: «Von Kopf bis Knie auf Circus eingestellt» (natürlich mit zwei Cs geschrieben). Seine Liebe zum Circus liess ihn in magischen

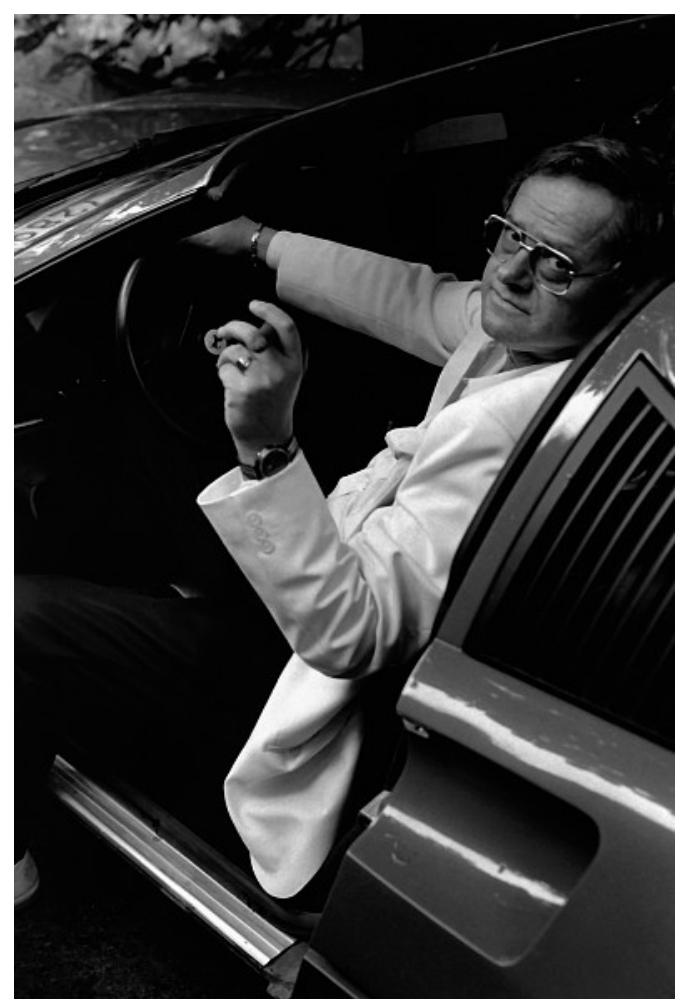

Hermann Burger im Ferrari.

Foto: Yvonne Böhler.
Zirkeln verkehren. Braucht erwähnt zu werden, dass Hermann Burger ein Wortgewaltiger, ein Wortjongleur war, wie er einzigartiger nicht sein kann? Bewusst war er sich dabei, dass jeder Akrobatik der Absturz droht.

Sind wir nun also am Ende des Rundganges angekommen? «Drei hohe Cs bestimmten mein Leben.»Wir erinnern uns dabei auch an die hohen Cs in der tenoralen Gesangskunst: Es ist das Höchstmass an Artistik (und an Sturzgefahr). Nein: Das Ende sieht ganz anderes aus: Des Autors Leben war ein Krankheitsleben. Bereits mit 30 Jahren begann seine manisch-depressive Erkrankung, die ihn zeitlebens begleitete wie die Psychiatrie. Seine Cyclothymie (das vierte C) ist es, die ihm den Tractatus logico-suicidalis (in Anlehnung an Wittgenstein und an Amérys «Hand an sich legen») diktiert. Die Depression, so Burger, ist Tod mit offenen Augen, doch anders als der Tod hat die Depression einen Stachel. Sein Leben werde, wie er in «Brunsleben» vorausahnend schreibt, mit 46 Jahren der infausten Diagnose (!) wegen zur Engführung. Enger, schliesslich, ging es nicht mehr. Seine oft todwitzigen Fiktionen werden im Wissen um dieses Ende zu todernsten Texten. Hier hilft kein Zaubertrick mehr, Hermann Burgers Autoeskamotage (sich selber wegzaubern) entbehrt jetzt jeden Spasses. Hermann Burger ist das Zentralmassiv der Depression: Sein Werk ist und war das Werk eines Toten. Er verfiel zunehmend der schwarzen Magie. Die Heilung, die Hermann Burger durch seine Literatur zu finden meinte, die Wiedergeburt in der «Künstlichen Mutter», ist eine Geburt zur makellosen leeren Existenz, eine Geburt zum Tode. Er, sagte er, habe keine Wahl, sein Leiden sei nicht heilbar, «das macht mich in mir selber unlebbar». Das Todespathos in seinen Werken, das oft ins Groteske umschlägt, weicht bitterstem Ernst. Der Traum vom Glück hiess für ihn unmissverständlich: geliebt werden, ohne davon sprechen zu müssen. Kurz vor seinem Tod hat er seinen Freunden einen langen Brief geschrieben. Da steht unter anderem: «Geht nicht fort, bleibt noch eine Weile ...» Wobei wir wieder bei Genialität, Manie und Depression angekommen wären. Am Schluss finden sich seine Sätze als Vermächtnis: «Meine gänzliche Verlassenheit in Mutterfinsternis.» Einen Endogendepressiven, hat Hermann Burger gesagt, sollte man jeden Tag öffentlich ehren, an dem er sich nicht das Leben genommen hat. Statt dessen wartet man, bis er es tut und kondoliert ... 